\title{
Management of strut graft failure in anterior cervical spine surgery
}

\author{
Issada Thongtrangan, M.D., Raju S. V. Balabhadra, M.D., and Daniel H. Kim, M.D. \\ Department of Neurosurgery, Stanford University Medical Center, Stanford, California
}

\begin{abstract}
Although successfully used, long strut grafts are vulnerable to dislodgment, displacement, fracture, and nonunion, which can require revision surgery; thus, meticulous preparation of the vertebral endplate along with exact sizing and harvesting of the bone graft with plating are essential for successful outcomes. Biomechanical data and previous clinical studies support the addition of posterior fusion and fixation following multilevel (more than two-level) corpectomy. The additional posterior instrumentation moves the instantaneous axis of rotation posteriorly, thus approximating its normal location in the posterior vertebral body (VB). Biomechanically, this protects the graft from excessive loads while in extension and explains the clinical success of circumferential instrumentation for long-segment corpectomy reconstructions. If strut fracture occurs with minimal displacement and the graft position is still satisfactory, application of a halo vest and judicious observation are recommended. Significant displacement, kyphosis, or loss of contact of the graft and VB require revision surgery. In patients requiring revision surgery for nonunion, placement of fibular autograft or allograft with use of bone morphogenetic protein is likely to be beneficial. If questions remain regarding bone quality or construct stability, the supplemental use of posterior stabilization is recommended. Various surgical approaches have been advocated for treatment of symptomatic anterior cervical pseudarthroses or nonunion. It remains controversial as to whether the anterior or posterior approach is best. Adequate understanding of the graft and implant biomechanics are essential for a successful outcome.
\end{abstract}

\section{KEY WORDS - fibular allograft - cervical corpectomy - vertebrectomy • failed allograft - revision spine surgery}

Multilevel involvement of the cervical spine by degenerative disease, tumors, trauma, or infection is not uncommon and presents several management challenges. With simple discectomy, spinal canal and foraminal decompression can be difficult, inadequate, or both because of the exposure being limited to the intervertebral space. Thus, long segment decompression by corpectomy and reconstruction with instrumentation is currently favored over multilevel discectomy and fusion. A corpectomy allows a wider neural decompression to be performed safely and under the surgeon's direct vision. Long strut grafts are known, however, to be vulnerable to graft migration, displacement, angulation, fracture, nonunion, and instrumentation failure requiring revision of the strut graft. The aim of this paper is to review strut graft failure with regard to its various causes, biomechanics, and management strategies.

\section{Causes of Strut Graft Failure}

High complication rates of up to $60 \%$ have been reported when performing multilevel cervical corpectomy and reconstruction. ${ }^{16,39}$ Graft dislodgment is reported in 5 to

Abbreviations used in this paper: $\mathrm{ACDF}=$ anterior cervical discectomy and fusion; $\mathrm{CT}=$ computerized tomography; $\mathrm{MR}=$ magnetic resonance; $\mathrm{VB}=$ vertebral body.
$50 \%$ of multilevel corpectomy patients when stand-alone grafts have been placed without plating. ${ }^{9,39}$ Thirty to $50 \%$ of the complications in these multilevel cases are due to graft- and instrumentation-related causes and carry a significant reoperation rate. ${ }^{9,16,34,39}$ The various causes of strut graft failure are summarized in Table 1. In an initial study, Zdeblick and Bohlman ${ }^{39}$ reported a $33 \%$ construct failure rate when using fibular allograft and all their patients required reoperation for graft extrusion. Vaccaro, et al., ${ }^{34}$ noted similar results, with a $9 \%$ failure rate in patients undergoing two-level anterior corpectomy and fusion with plating and a $50 \%$ failure rate for three-level anterior corpectomy and fusion with plating.

Sasso, et al., ${ }^{30}$ reported a $6 \%$ failure rate in their patients who underwent fixed-plated cervical spine locking plate two-level anterior cervical corpectomy and fusion, but a $71 \%$ failure rate after three-level fixed-plated anterior cervical corpectomy and fusion. Five of seven patients who underwent a three-level anterior corpectomy and fusion experienced construct failure within 2 months after surgery. All five failures were catastrophic graft/plate displacements, with the graft usually cavitating into the caudal vertebra and "kicking out" of the graft/plate anteriorly. Three of these patients underwent revision surgery consisting of posterior cervical fusion with lateral mass instrumentation. Of three patients, two were found to have a delayed fracture of the strut graft after healing of the 
TABLE 1

Causes of strut graft failure following anterior cervical fusion

graft related

graft angulation

graft displacement

graft fracture: early or delayed

subsidence

nonunion

instrumentation failure

screw pullout/fracture

plate fracture

host factors

poor bone quality: osteoporosis

extreme kyphosis

long segment grafting (three or more levels)

inadequate correction of kyphotic deformity or subluxation

fusion extending into cervicothoracic junction

technical factors

short/long graft

improper preparation of fusion bed

faulty choice of surgical procedure

graft-host junctions. Thus, the complication rate is often associated with the number of levels of corpectomy and the length of the strut graft.

Comparing the use of iliac crest autograft and freezedried iliac graft, Zdeblick and Ducker ${ }^{40}$ showed the nonunion rate to be significantly higher in the allograft group (62\%) compared with the autograft group (17\%) in multilevel fusions, although the nonunion rate was identical in single-level fusions (5\%). Fernyhough, et al., ${ }^{15}$ reported a $27 \%$ nonunion rate with fibular autograft compared with a $41 \%$ nonunion rate with fibular allograft used to stabilize the multilevel cervical spondylotic spine following multilevel discectomy and corpectomy.

\section{Biomechanical Considerations}

Anterior Strut Grafting and Instrumentation. Biomechanical data and previous clinical studies support the addition of posterior fusion for added stabilization of plated multilevel anterior cervical fusions. ${ }^{13,25}$ DiAngelo, et al.,${ }^{9}$ and Foley, et al., ${ }^{16}$ have demonstrated that the instantaneous axis of rotation is shifted anteriorly after the application of an anterior plate. The result is a reversal of the loading pattern in a long strut graft so that no loading of the graft occurs during flexion moments and a profound excessive compression of the graft occurs during extension load. Thus, anterior cervical plating may promote "pistoning" of the graft through the caudal vertebral endplate and the plate subsequently kicking out of the lower VB. The addition of posterior instrumentation moves the instantaneous axis of rotation posteriorly, thus approximating its normal location in the posterior aspect of the VB. Biomechanically, this protects the graft from excessive loads during extension and explains the clinical success of circumferential instrumentation for long-segment corpectomy reconstructions. Isomi, et al. ${ }^{21}$ and Panjabi, et al., ${ }^{26}$ evaluated the use of the anterior cervical plate in a single-level and three-level corpectomy model reconstructed with a strut graft without a posterior implant. After 1000 cycles of fatigue, they noted instability and significantly increased motion at the inferior end of the plate in the three-level corpectomy. The results of these studies underline the significance of an additional posterior implant in a three-or-more level corpectomy.

Longer plate fixations create longer lever arms, placing higher stress on the caudal screws; thus, longer plate fixations may still require halo vest immobilization, especially in patients who are unreliable, noncompliant, exhibit a propensity to fall because of their neurological deficit, or have poor bone quality.

Types of Plating Systems. The type of anterior plate, dynamic compared with rigid, may affect the success of the anterior-only construct. Anterior plating should loadshare with the graft to facilitate fusion as well as to protect the graft from high stresses that may cause graft collapse. Rigid plating systems, which resist any settling of the construct, may hold the construct in some distraction and delay or prevent fusion. Recently, two dynamic plating systems, the DOC plate (Depuy-Acromed, Cleveland, $\mathrm{OH}$ ) and the ABC plate (Aesculap, Bethlehem, PA) became commercially available. These are load-sharing plating systems designed to protect the graft from high loads, which can cause graft collapse. At the same time, they allow controlled subsidence of the construct as graft settling occurs in the postoperative period, thus preventing instrumentation failure often seen in long segment strut grafts.

Brodke and colleagues (Cervical Spine Research Society Meeting, 1999, unpublished data) demonstrated a comparative biomechanical study of the DOC, ABC, CSLP (Synthes Spine, Paoli, PA), and Orion plates (Medtronic Sofamor-Danek, Memphis, TN) in a $30-\mathrm{mm}$ corpectomy model. The results showed that the DOC plate and $\mathrm{ABC}$ plate were able to load-share effectively with the $30-\mathrm{mm}$ graft and the $27-\mathrm{mm}$ graft with a $10 \%$ subsidence rate for each. The static plates were unable to load-share with the 27-mm strut graft. They concluded that the DOC system was significantly less stiff in all testing modes compared with the $\mathrm{ABC}$ plate and the constrained plates.

In a clinical study, Apfelbaum and colleagues (Cervical Spine Research Society Meeting, 1999, unpublished data) reviewed the $\mathrm{ABC}$ system results in 149 patients who underwent corpectomy or ACDF. Corpectomies were performed in 24\%, whereas ACDF was performed in $76 \%$. Single-level plates were used in $43 \%$, two-level plates in $44 \%$, and three-or-more level plates in $13 \%$. Allograft bone was used in 56\% and autograft bone in $44 \%$. Fusion was reported in $70 \%$ of levels at 3 months and $86 \%$ at 6 months. There were no hardware-related failures or symptomatic nonunions. Subsidence of 1 to $2 \mathrm{~mm}$ was noted in most constructs and was more prevalent when allograft was used. Most of the settling occurred within the 1st month after surgery.

Junctional plating is a technique in which a small anterior cervical plate is fixed at one end of the construct, usually at the lower vertebra graft since dislodgment often occurs at the inferior end of the construct. It overlaps the end of the strut graft-vertebra junction and is able to block the end of the graft so that it does not dislodge anteriorly. It has the advantage of not creating a tension band anteriorly, which can lead to large stresses on the strut graft. Unlike rigid plating systems, it does not act as a distraction device. Instead, it allows load-sharing by the graft and permits the graft to settle gradually into the endplates, 
which is necessary for the graft to achieve a solid arthrodesis. ${ }^{28}$ Vanichkachorn, et al. ${ }^{35}$ reported on 11 patients treated for cervical myelopathy with an average of 3.36 levels undergoing corpectomy. The corpectomies were reconstructed with fibular or iliac crest strut grafts and junctional plating. All patients underwent additional posterior segmental fixation. No patient in this series experienced graft- or construct-related complications.

Although there are no prospective studies in which the outcomes of rigid and dynamic plating for multilevel corpectomy and reconstruction are compared, dynamic plating is likely to be more effective, because it facilitates fusion by load-sharing and permits subsidence seen with long grafts, thus avoiding instrumentation failure. There is a significant biomechanical advantage to placing a posterior segmental fixation in a long segment corpectomy of more than two levels.

\section{Clinical Symptoms}

The clinical presentation depends on the cause of the strut graft failure. Many patients with nonunion remain asymptomatic. In patients with symptomatic nonunion, pain is the most common presenting symptom. Lowery, et al. ${ }^{24}$ suggested that continued or worsening axial pain 6 months after the initial procedure and positive radiographic images are consistent with nonunion and pseudarthrosis. Several recent studies with long-term follow up support the relationship between pseudarthrosis and a poor outcome. Bohlman, et al., ${ }^{3}$ demonstrated the relationship between postoperative neck or arm pain and pseudarthrosis after long-term follow up in patients, who underwent ACDF. They concluded that motion and presence of chondroosseous spurs at the level of the pseudarthrosis may contribute to residual nerve root compression. Phillips and colleagues (North American Spine Society Meeting, 1996, unpublished data) reported that among their patients with symptomatic pseudarthroses, one third had a prolonged symptom-free interval following initial surgery, despite radiographic evidence of pseudarthrosis, and developed symptoms only after a subsequent traumatic event. This finding suggests disruption of a fibrous union as the cause of symptoms.

Nonunion in the presence of an anterior cervical fixation device carries the additional risk of screw fracture or migration with associated esophageal penetration, mediastinitis, and death. ${ }^{33,38}$ The diagnosis and treatment of such a nonunion should be pursued aggressively. Patients with early- or late-onset graft fracture usually present with neck pain.

Patients with anterior graft dislodgment may present with difficulty swallowing or breathing. With posterior graft displacement, the patient may present with new-onset neurological deficits.

\section{Neuroimaging Investigation}

Radiographic criteria used to assess strut graft fusion include the presence of bone trabeculae crossing the disc spaces, absence of radiolucent lines at the graft-host junction and less than $2 \mathrm{~mm}$ of motion between adjacent spinous processes on flexion-extension views. Cannada, et al.,$^{5}$ concluded that measurement of the change in distance between the spinous processes is more reproducible and accurate than the Cobb method for making the diagnosis of pseudarthrosis. Subsidence of the graft can be observed on the sequential radiographic images. Failure of the implant as manifested by loosening and/or breakage of screws and broken plates implies the possibility of pseudarthrosis because the majority of the load will be transferred to the implant, which then leads to fatigue failure. Graft fracture, malposition, and dislodgment are obvious evidence of failure of the construct. Sequential standard $\mathrm{x}$-ray films including flexion-extension views are mandatory for the assessment of strut graft failure.

Tomography, CT, and scintigraphy scanning may be necessary in questionable cases. Tomography increases the accuracy of diagnosis to $96 \% .^{8}$ Single-photon emission CT scanning involves the use of a tomographic camera to remove three-dimensional superimposition from scintigraphic images, thereby improving image contrast and offering more complete spatial information than conventional bone scans. It is increasingly being used to demonstrate increased focal uptake at sites of pseudarthrosis. ${ }^{32}$

Computerized tomography scanning has proved useful in the diagnosis of pseudarthrosis. Direct coronal imaging and the use of three-dimensional artifact CT scanning have been shown to improve the rate of diagnosis over conventional $\mathrm{CT}^{41}{ }^{41}$ Epstein $^{12}$ demonstrated the valuable use of two- or three-dimensional CT in the diagnosis of nonunion, pseudarthrosis, and graft fracture.

\section{Management of Established Strut Graft Failure}

Fracture and Dislodgment. Bone graft-related complications in the early postoperative period include graft fracture, collapse and dislodgment, and donor site-related problems. Graft fracture is also more likely when the graft is harvested with an osteotome instead of a saw. ${ }^{22} \mathrm{~A}$ fracture of a Robinson-type graft may still heal and may be observed if radiculopathy does not recur and no significant kyphosis results. If the bone is minimally displaced and is in a satisfactory position, the application of a halo vest and judicious observation can be attempted. In contrast, doweltype anterior grafts are weaker in compression and may collapse, resulting in a kyphotic deformity and possibly neural deficit; revision of the strut graft is likely to be required. ${ }^{36}$ Patients with significant fracture displacement require a revision of the strut graft and instrumentation or additional posterior fusion and fixation.

Compared with small grafts used for fusion following discectomy, long anterior strut grafts are more vulnerable to dislodgment, and careful technique in preparing the VB endplate is essential. The aggressive removal of the bone endplate is avoided to prevent graft migration into the VB. The ends of the strut are rounded with a high-speed burr. The superior and inferior vertebral recipient sites are prepared with the burr to match the ends of the graft. The anterior and posterior vertebral cortical ridges are preserved to prevent extrusion or posterior migration of the strut graft after placement. The graft is placed into the trough and the ends are countersunk into position before the intervertebral distractor is released. Gentle flexion-extension movements of the neck are performed before plating to confirm that the graft is locked in a stable position. When using a fibular strut, its flattest surface should face the floor of the canal, even if this necessitates narrowing 
its dimensions slightly with the high-speed burr. This prevents the chance of encroachment of the graft into the spinal canal. Local bone graft from the corpectomy is morselized and placed inside the fibular graft to enhance graft incorporation. Graft position and operative levels must be confirmed radiographically before the patient leaves the operating room.

In patients with osteoporosis, the VB into which the graft is seated can fracture. If this occurs and the fracture is minimally displaced and the graft position is still satisfactory, the application of a halo vest (if not already in place) and close observation are recommended. If no further displacement occurs and there is no kyphosis, the fracture is likely to heal and to yield a good result. Significant displacement, kyphosis, or loss of contact of the graft and VB requires revision surgery. This usually means obtaining a longer graft and extending the fusion one more level. If questions remain regarding the quality of the bone or stability of the construct, supplemental posterior stabilization is recommended. ${ }^{11}$

In long-segment fibular grafts, the process of fusion often takes 1 to 2 years and occurs by "creeping substitution," beginning at the endplates and extending toward the midportion of the strut graft. As bone resorption precedes new bone formation, however, the strut grafts are relatively weak and are susceptible to delayed fracture with normal loading of the cervical spine when external bracing is discontinued. These are often successfully managed with additional posterior fusion and instrumentation.

Several techniques may be used for posterior cervical fusion. As stated, in the presence of intact posterior elements and good-quality host bone, interspinous wiring will usually suffice. In the absence of posterior elements or when faced with significant osteopenia or hypoplastic spinous processes, one should consider using lateral mass plate/screw fixation. Lateral mass plating techniques afford excellent immediate stability, particularly in multilevel procedures. The technique, however, should be performed only after one becomes thoroughly acquainted with the local anatomy and the potential difficulties associated with the procedure. ${ }^{1,10,18-20}$ Longer plate fixations create longer lever arms, placing higher stress on the caudal screws, and the risk of screw avulsion must be kept in mind. It is important to consider the use of halo vest immobilization in patients who are unreliable or noncompliant, exhibit a propensity to fall because of their neurological deficit, or have impaired healing potential.

Nonunion. Various surgical approaches have been advocated for the treatment of symptomatic anterior cervical pseudarthroses or nonunion. It is still controversial as to whether the anterior or posterior approach is the appropriate procedure. Several reports in the literature support both approaches. In patients requiring revision surgery for nonunion, fibular autograft may be considered, especially in those who smoke cigarettes. Alternatively, the use of fibular allograft with the use of bone morphogenetic protein is recommended to improve fusion rates. Coric, et al., ${ }^{7}$ recommended the anterior approach for reopening of the pseudarthrosis, fusion with allograft, and plate stabilization of the segment, with a $100 \%$ fusion rate and no significant complications. Caspar, et al., ${ }^{6}$ performed revision surgery through an anterior approach by using a trape- zoidal plate in 37 patients in whom anterior pseudarthrosis was diagnosed. Of 37 patients 21 experienced complete alleviation of head-neck-shoulder pain, 10 improved, and six remained unchanged. Radiographic images revealed a stable bone fusion in optimal alignment in all 37 patients.

Because of the technical considerations of repeated anterior surgery, posterior cervical fusion to correct an anterior nonunion was proposed initially by Riley, et al. ${ }^{29}$ The additional posterior stabilization encourages the anterior fusion mass to consolidate. Posterior revision surgery was noted to be successful in achieving bone union 94 to 100\% of the time. . $^{4,14,23,31}$

Farey, et al., ${ }^{14}$ reported on 19 patients with symptomatic anterior pseudarthroses and radiculopathy. These patients underwent a posterior nerve root decompression, posterior arthrodesis with autograft, and triple-wire stabilization. A solid posterior fusion was noted in $100 \%$ of the patients. Consolidation of the anterior pseudarthrosis was achieved in $100 \%$. An excellent clinical outcome result was accompanied by fusion success in $95 \%$ of the patients. Lowery, et al. ${ }^{24}$ in a retrospective study, demonstrated the efficacy of the posterior lateral mass plate in patients who suffered from symptomatic pseudarthrosis after revision surgery. Twenty patients underwent anterior nonunion resection and regrafting with anterior instrumentation and solid fusion was achieved in only $45 \%$. Seventeen procedures were performed using a posterior approach, including posterior cervical fusion and articular pillar plating. Of these 17 patients, solid fusion was achieved in 16. Circumferential procedures were performed in seven patients and solid fusion was achieved in all patients. They concluded that posterior cervical articular mass/pillar plating and fusion resulted in a higher probability of fusion than repeated anterior procedures, even with the addition of anterior plate stabilization. Posterior fusion and articular plating, whether alone or part of a circumferential procedure, provides the added fixation required to repair successfully failed anterior cervical fusions.

Titanium mesh cages have been widely used for spinal reconstruction since 1986 when they were first introduced. Despite their popularity, relatively few studies have been published on their use in the cervical spine. These cages can be used as structural devices containing autologous local bone or iliac crest bone graft, obviating the need to harvest large structural bone grafts. The disadvantages of their use are the increased cost and the difficulty of assessing fusion status. Riew and Rhee, ${ }^{27}$ reported on 54 patients who underwent placement of titanium mesh cages as part of their corpectomy reconstruction. No instance of extrusion, infection, neurological deficit, or graft collapse occurred during the follow-up period. The fusion rate is similar to those achieved with autograft and allograft. Only two patients have required revision surgery for pseudarthroses; both underwent multilevel procedures and were cigarette smokers. Hee, et al., ${ }^{17}$ reported on 21 patients who underwent multilevel cervical corpectomies and reconstruction with titanium cages and anterior plating. The major complications (33\%) are largely the result of subsidence and instrumentation failures, especially in patients with osteopenic bone; thus, although titanium mesh cages can be used as an alternative to strut grafts to avoid the harvesting of the grafts, their use should be 
avoided in patients with osteopenia. Furthermore, they are not designed to be used as stand-alone devices. They must be used in combination with anterior plating, with or without additional posterior fixation.

\section{Strut Graft Techniques Following Corpectomy}

Iliac Crest Strut Graft. Tricortical iliac crest graft provides more cancellous bone, which may facilitate an earlier successful fusion. It is associated, however, with donor-site morbidity and the natural curvature of the iliac crest makes it unsuitable for spanning a distance of more than two VBs. Tricortical iliac crest graft is recommended for one- or two-level corpectomies because the iliac crest configuration fits well with the contours of the cervical spine. Surgeons should prepare this graft to fit snugly at both ends of the corpectomies to avoid failure and complications. Many surgical techniques have been published.

The Bohlman Technique. Following decompression skeletal traction is increased to between 30 and $40 \mathrm{lbs}$. This assists in the correction of the deformity and in the seating of the bone graft. Seating holes $5 \mathrm{~mm}$ deep are prepared in the central endplate of the adjacent VB by using a high-speed burr. The length of the graft is estimated using calipers or a malleable probe. A T-shaped tricortical iliac crest strut graft is harvested and placed with the three-sided cortex posteriorly or anteriorly (Fig. 1). The former provides a smooth posterior edge and biomechanically provides greater strength and axial loading. The graft is impacted into the superior vertebral seating hole with a bone graft tamp following which the graft is countersunk into the inferior vertebral seating hole. Traction is reduced to $10 \mathrm{lbs}$ and graft stability is tested using a Kocher clamp. The traction may be removed and stability tested by cervical flexion and extension. Additional pieces of cancellous bone are placed around the ends of the bone graft. The anterior aspect of the bone graft must be

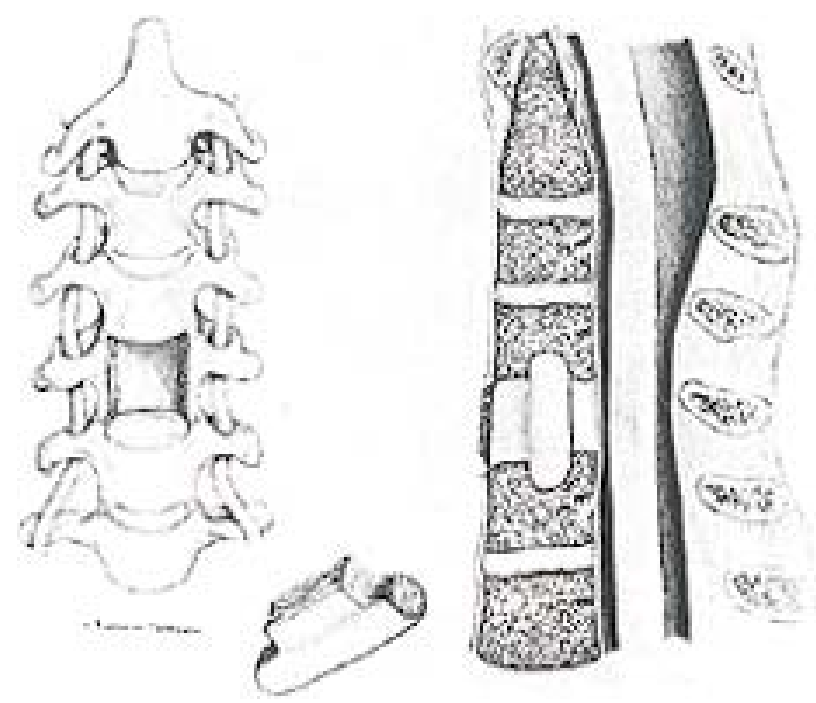

Fig. 1. Artist's illustrations of an iliac crest strut graft, with cortical surface faced anteriorly. An angled burr was used to prepare the superior and inferior end plates by creating the shallow seating holes to accept and lock the graft in place. Reprinted with permission from Lippincott Williams \& Wilkins. smoothed with a burr to prevent esophageal abrasion. An intraoperative radiograph is obtained to ensure satisfactory graft position and spinal alignment. ${ }^{2}$

Fibular Strut Graft. Fibular allograft is indicated and most useful in multilevel corpectomies. If autograft is used, the fibular graft must be harvested from the middle third of the fibula to prevent derangement of normal ankle biomechanics. The superficial branch of the common peroneal nerve must be identified and protected. The ends of the fibular graft are fashioned with a high-speed burr to fit the prepared seating holes in the VBs at either end of the decompression. The broad aspect of the fibula is placed posteriorly during insertion. There are several techniques recommended in the literature.

The Whitecloud/LaRocca Graft Technique. Following decompression, the superior and inferior VBs are notched anteriorly. Notches are cut on both ends of the graft; those on the end to be inserted superiorly are of equal length, but the posterior extension of the inferior notch is slightly shorter than the anterior one, which allows for easier insertion. The cartilaginous endplates of the inferior and superior aspects of the VBs to be fused are removed. Seating holes are then cut into the end plates of the superior and inferior VBs with the high-speed burr. The graft is sculpted so that it is rounded at its superior end and then driven into the vertebra below with impaction. When traction is released, the graft is locked into position. The graft is inserted with traction applied to the cervical spine and locked into place when traction is discontinued. The graft configuration prevents both posterior migration into the spinal canal and anterior extrusion. Once in place, two thirds of the graft is deep in the anterior aspect of the vertebral column (Fig. 2). ${ }^{37}$
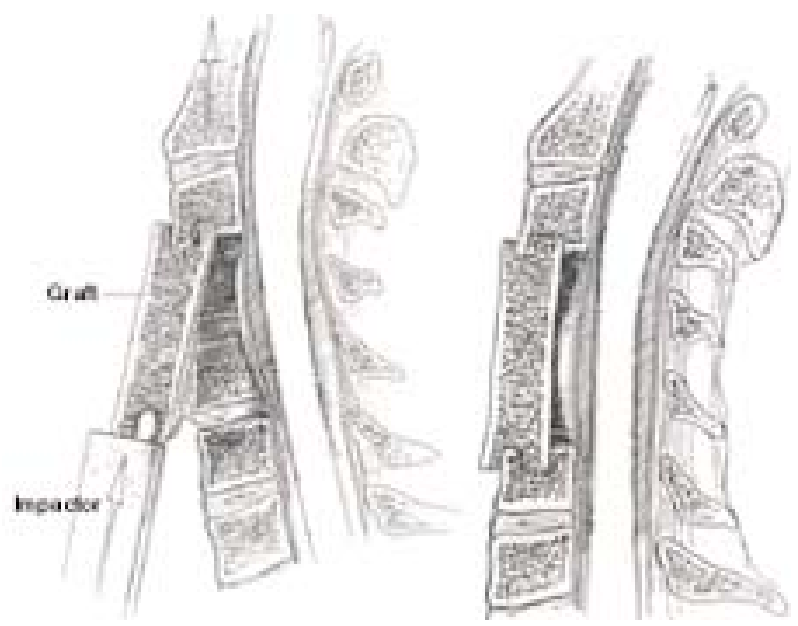

Fig. 2. Artist's illustrations of a fibula strut allograft used in the Whitecloud/Larocca technique. The allograft fibula is prepared by notching each end with a high-speed burr. The graft is gently impacted superiorly. While traction is applied to the neck, the inferior portion is gently tamped posteriorly into place. Notching allows the graft to be locked into the anterior cortices of the superior and inferior VBs. Care must be taken to ensure that the width of the end plate holes is wide enough to accept the notched portions of the fibula. Reprinted with permission from Lippincott Williams \& Wilkins. 
The Zdeblick/Bohlman Graft Technique. Zdeblick and Bohlman reported on a similar procedure, but modified the technique for insertion of the fibular graft by drilling troughs for the graft in the cortical end plates of VBs adjacent to the area of decompression and inserting the fibular strut into the prepared troughs (Fig. 3). The graft is fashioned as a peg to fit snugly into the adjacent VB seating holes. The superior portion of the graft is positioned first. Cranial skeletal tong traction is increased to between 20 and $30 \mathrm{lbs}$. The inferior portion of the graft is then gently tapped into the position. Traction is released after positioning of the graft. ${ }^{39}$

\section{ILLUSTRATIVE CASE REPORT}

This 62-year-old woman had suffered from C4-6 myelopathy for several years. She underwent a total C-4 vertebrectomy and partial C-3 and C-5 fibular strut allograft fusion at another institution. No internal stabilization was performed, but the patient was instructed to wear a cervical brace. Unfortunately, 3 months after the surgery the patient gradually developed quadriparesis, axial neck pain, and difficulty swallowing. This patient was referred to our institution for revision surgery. A lateral x-ray film (Fig. 4) of the spine showed complete dislodgment of the fibular allograft at the lower junction and kyphotic deformity. A sagittal $\mathrm{T}_{2}$-weighted $\mathrm{MR}$ image of the cervical spine revealed dislodgment of the graft, signal changes in the spinal cord at that level, and a kyphotic deformity of the cervical spine (Fig. 5). We performed revision surgery proceeding with combined anterior and posterior procedures. The failed bone graft was removed anteriorly and further corpectomy of C-3, C-4, and C-5 for decompres-
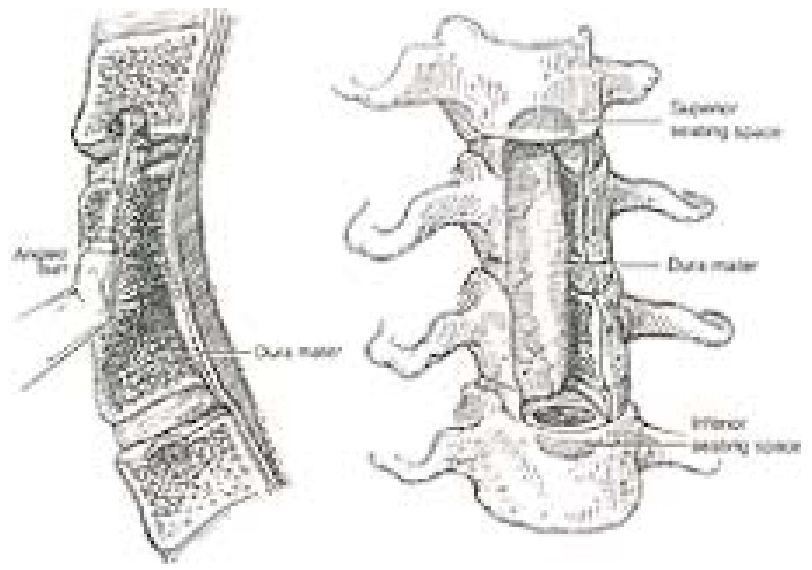

Fig. 3. Illustrations showing a fibula strut allograft used in the Zdeblick/Bohlman technique. An angled burr is used to prepare the superior and inferior end plates by creating shallow seating holes to accept the graft. Care is taken to avoid violation of the anterior or posterior cortices. The depth of the seating holes should be less than half the height of the VB. The inferior seating hole may be fashioned in a manner identical to that used for the superior seat. Occasionally an inverted L-shaped trough may be used for ease of placement of the inferior portion of the graft, avoiding traumatic disruption of the anterior cortical rim as the graft is tamped into place. Reprinted with permission from Lippincott Williams \& Wilkins.

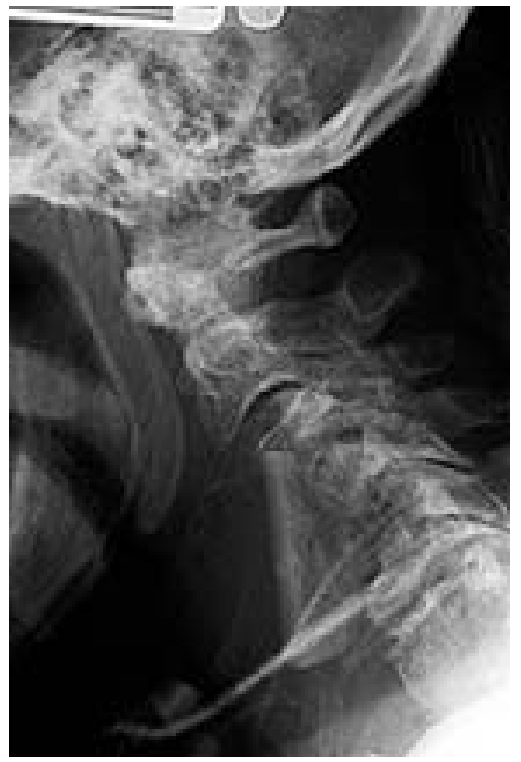

Fig. 4. Lateral $\mathrm{x}$-ray film of the cervical spine demonstrating failed strut allograft following anterior cervical corpectomy without fixation.

sion of the residual stenosis and the fibular graft was prepared in such a way that it locked from C-2 to C-6. Anterior plating was placed from C-2 to C-6 and additional posterior fixation was essential in this patient because the length of the construct was more than three levels. Lateral mass screws and plate were placed from C-3 to C-6. A postoperative lateral view of the cervical spine revealed that the alignment of the cervical spine was maintained and the fibular allograft spanned from C-2 to C-6

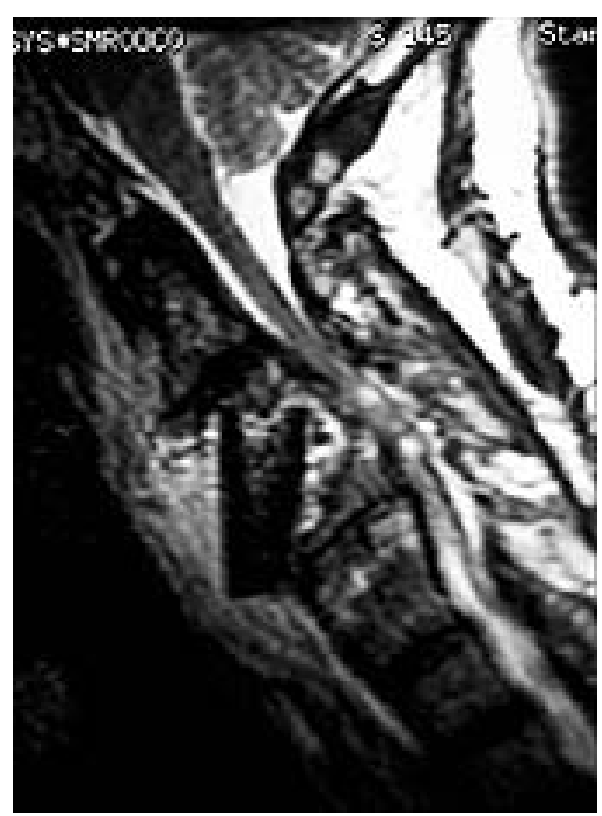

Fig. 5. Sagittal $\mathrm{T}_{2}$-weighted MR image revealing complete dislodgement of the graft at the lower end and canal impingement with signal changes of the spinal cord. 


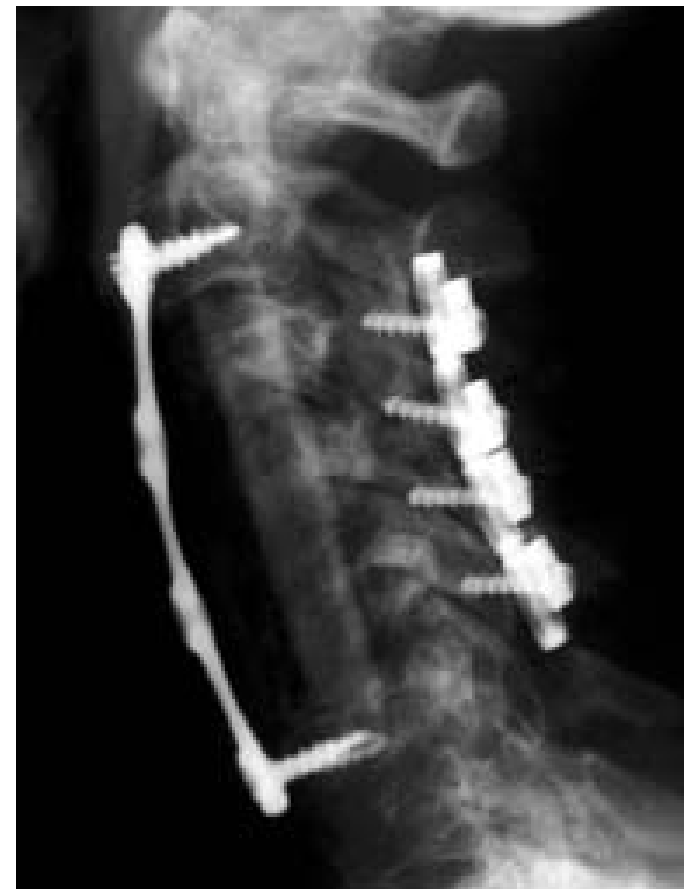

Fig. 6. Lateral x-ray film obtained after revision surgery revealing revision of the fibular strut allograft and combined anterior and posterior plating.

with anterior and posterior plating (Fig. 6). A postoperative sagittal $\mathrm{T}_{2}$-weighted MR image of the cervical spine revealed some metal artifacts, but it clearly demonstrated that there was an improvement in the signal changes of the spinal cord (Fig. 7).

\section{DISCUSSION}

Multilevel corpectomies and interbody grafting and corpectomies pose a more challenging environment for graft incorporation. Although autografts are more successful, long-segment harvesting is associated with significant donor site complications and morbidity. If allograft is preferred, the use of the fibular strut graft showed reliable results. The use of the fibular graft has several advantages: it is strong and permits a graft of any length and it provides a central cavity for the packing of autologous cancellous bone harvested from the corpectomy site to enhance the fusion rate. Anterior plating improves the initial stability after corpectomy and strut grafting and reduces the risks of graft dislodgment. Meticulous attention to proper graft placement is crucial. If the plate is not properly contoured, it can act as a distraction device, can prevent intimate bone contact between the graft and vertebral endplate, and can inhibit healing. Vaccaro, et al., ${ }^{34}$ and Sasso, et al., ${ }^{30}$ reported a high rate of failure in patients with three- and four-level spondylotic disease treated with strut grafting and plating. If a long strut allograft (more than two-level corpectomy) is used, adjuvant posterior stabilization should be considered. We do not recommend screw fixation into the allograft itself because of the increased rate of fracture of the graft. In symptomatic nonunion, use of fibular autograft or allograft with use of bone

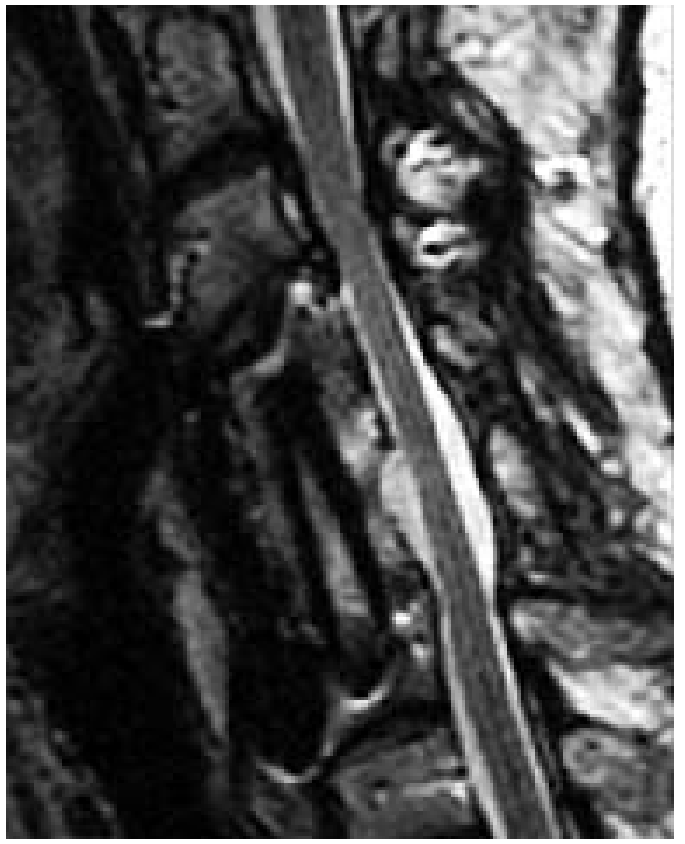

Fig. 7. Postoperative sagittal $\mathrm{T}_{2}$-weighted MR image demonstrating good alignment without cord impingement and improvement of spinal cord signals.

morphogenetic protein is likely to be beneficial. Although the titanium mesh cage filled with allograft and/or combined with cancellous autograft from iliac crest or VB is another alternative for long strut allograft or failed long strut allograft, it should be avoided in patients with poorquality bone. Furthermore, it is not designed to be used as a stand-alone device.

\section{CONCLUSIONS}

Prevention is better than treatment of the established strut graft failure. The surgeon should perform a delineated surgical procedure to prevent failure of the strut graft. Understanding the biomechanics of the graft as well as the implant is crucial. Regardless of graft source, meticulous preparation of the bone graft-host bone interface and secure placement of the graft with plating is essential for success of long-segment anterior cervical fusions. Anterior plating is a reliable construct for fewer than two-level corpectomies. The addition of a posterior construct is essential in three- or multilevel corpectomies. Dynamic plating systems are likely to be more beneficial than rigid plating systems by permitting load-sharing by the graft together with controlled graft subsidence, and thus avoiding instrumentation failure.

\section{References}

1. An HS, Gordin R, Renner K: Anatomic considerations for plate-screw fixation of the cervical spine. Spine 16 (Suppl 10): S548-S551, 1991

2. Bohlman HH, Eismont FJ: Surgical techniques of anterior decompression and fusion for spinal cord injuries. Clin Orthop 154:57-67, 1981 
3. Bohlman HH, Emery SE, Goodfellow DB, et al: Robinson anterior cervical discectomy and arthrodesis for cervical radiculopathy. Long-term follow-up of one hundred and twenty-two patients. J Bone Joint Surg Am 75:1298-1307, 1993

4. Brodsky AE, Khalil MA, Sassard WR, et al: Repair of symptomatic pseudarthrosis of anterior cervical fusion. Posterior versus anterior repair. Spine 17:1137-1143, 1992

5. Cannada LK, Scherping SC, Yoo JU, et al: Pseudoarthrosis of the cervical spine: a comparison of radiographic diagnostic measures. Spine 28:46-51, 2003

6. Caspar W, Pitzen T: Anterior cervical fusion and trapezoidal plate stabilization for re-do surgery. Surg Neurol 52:345-352, 1999

7. Coric D, Branch CL Jr, Jenkins JD: Revision of anterior cervical pseudarthrosis with anterior allograft fusion and plating. $\mathbf{J}$ Neurosurg 86:969-974, 1997

8. Dawson EG, Clader TJ, Bassett LW: A comparison of different methods used to diagnose pseudarthosis following posterior spinal fusion for scoliosis. J Bone Joint Surg Am 67: 1153-1159, 1985

9. DiAngelo DJ, Foley KT, Vossel KA, et al: Anterior cervical plating reverses load transfer through multilevel strut-grafts. Spine 25:783-795, 2000

10. Ebraheim NA, Hoeflinger MJ, Salpietro B, et al: Anatomic considerations in posterior plating of the cervical spine. J Orthop Trauma 5:196-199, 1991

11. Emery SE, Bolesta MJ: Complications of cervical spine surgery, in Bridwell KH, DeWald RL (eds): The Textbook of Spinal Surgery, ed 2. Philadelphia: Lippincott-Raven, 1997, Vol. 2, pp 1427-1438

12. Epstein NE: Reoperation rates for acute graft extrusion and pseudarthrosis after one-level anterior corpectomy and fusion with and without plate instrumentation: etiology and corrective management. Surg Neurol 56:73-81, 2001

13. Epstein NE: The value of anterior cervical plating in preventing vertebral fracture and graft extrusion after multilevel anterior cervical corpectomy with posterior wiring and fusion: indications, results, and complications. J Spinal Disord 13:9-15, 2000

14. Farey ID, McAfee PC, Davis RF, et al: Pseudarthosis of the cervical spine after anterior arthrodesis. Treatment by posterior nerve root decompression, stabilization, and arthrodesis. J Bone Joint Surg Am 72:1171-1177, 1990

15. Fernyhough JC, White JI, LaRocca H: Fusion rates in multilevel cervical spondylosis comparing allograft fibula and autograft fibula in 126 patients. Spine 16 (Suppl 10):561-564, 1991

16. Foley KT, DiAngelo DJ, Rampersaud YR, et al: The in vitro effects of instrumentation on multilevel cervical strut-graft mechanics. Spine 24:2366-2376, 1999

17. Hee HT, Majd ME, Holt RT, et al: Complications of multilevel cervical corpectomies and reconstruction with titanium cages and anterior plating. J Spinal Disord Tech 16:1-9, 2003

18. Heller JG: Posterior cervical plate fixation: the concerns, in Garfin SR, Northrup BE (eds): Surgery for Spinal Cord Injuries. New York: Raven Press, 1993, pp 123-135

19. Heller JG, Carlson GD, Abitbol JJ, et al: Anatomic comparison of the Roy-Camille and Magerl techniques for screw placement in the lower cervical spine. Spine 16 (Suppl 10):S552-S557, 1991

20. Heller JG, Silcox DH III, Sutterlin CE III, et al: Complications of posterior cervical plating. Spine 20:2442-2448, 1995

21. Isomi T, Panjabi MM, Wang JL, et al: Stabilizing potential of anterior cervical plates in multilevel corpectomies. Spine 24: 2219-2223, 1999
22. Jones AA, Dougherty PJ, Sharkey NA, et al: Iliac crest bone graft. Osteotome versus saw. Spine 18:2048-2052, 1993

23. Lindsey RW, Newhouse KE, Leach J, et al: Nonunion following two-level anterior cervical discectomy and fusion. Clin Orthop 223:155-163, 1987

24. Lowery GL, Swank ML, McDonough RF: Surgical revision for failed anterior cervical fusions. Spine 20:2436-2441, 1995

25. McAfee PC, Bohlman HH, Ducker TB, et al: One-stage anterior cervical decompression and posterior stabilization. A study of one hundred patients with a minimum of two years of followup. J Bone Joint Surg Am 77:1791-1800, 1995

26. Panjabi MM, Isomi T, Wang JL: Loosening at the screw-vertebra junction in multilevel anterior cervical plate constructs. Spine 24:2383-2388, 1999

27. Riew KD, Rhee JM: The use of titanium mesh cages in the cervical spine. Clin Orthop 394:47-54, 2002

28. Riew KD, Sethi NS, Devney J, et al: Complications of buttress plate stabilization of cervical corpectomy. Spine 24: 2404-2410, 1999

29. Riley LH Jr, Robinson RA, Johnson KA, et al: The results of anterior interbody fusion of the cervical spine. Review of ninety-three consecutive cases. J Neurosurg 30:127-133, 1969

30. Sasso RC, Ruggiero RA Jr, Reilly TM, et al: Early reconstruction failures after multilevel cervical corpectomy. Spine 28: 140-142, 2003

31. Shinomiya K, Okamoto A, Kamikozuru M, et al: An analysis of failures in primary cervical anterior spinal cord decompression and fusion. J Spinal Disord 6:277-288, 1993

32. Slizofski WJ, Collier BD, Flatley TJ, et al: Painful pseudarthosis following lumbar spinal fusion: detection by combined SPECT and planar bone scintigraphy. Skeletal Radiol 16: 136-141, 1987

33. Smith MD, Bolesta MJ: Esophageal perforation after anterior cervical plate fixation: a report of two cases. J Spinal Disord 5:357-362, 1992

34. Vaccaro AR, Falatyn SP, Scuderi GJ, et al: Early failure of long segment anterior cervical plate fixation. J Spinal Disord 11: 410-415, 1998

35. Vanichkachorn JS, Vaccaro AR, Silveri CP, et al: Anterior junctional plate in the cervical spine. Spine 23:2462-2467, 1998

36. West CG: Bilateral brachial paresis following anterior decompression for cervical spondylosis. Spine 11:176-178, 1986

37. Whitecloud TS III, LaRocca H: Fibular strut graft in reconstructive surgery of the cervical spine. Spine 1:33-43, 1976

38. Yee GK, Terry AF: Esophageal penetration by an anterior cervical fixation device. A case report. Spine 18:522-527, 1993

39. Zdeblick TA, Bohlman HH: Cervical kyphosis and myelopathy. Treatment by anterior corpectomy and strut grafting. J Bone Joint Surg Ám 71:170-182, 1989

40. Zdeblick TA, Ducker TB: The use of freeze-dried allograft bone for anterior cervical fusions. Spine 16:726-729, 1991

41. Zinreich SJ, Long DM, Davis R, et al: Three-dimensional CT imaging in postsurgical "failed back" syndrome. J Comput Assist Tomogr 14:574-580, 199

Manuscript received July 28, 2003.

Accepted in final form August 29, 2003.

Address reprint requests to: Daniel H. Kim, M.D., Department of Neurosurgery, Stanford University Medical Center, Room R-201, Edwards Building, 300 Pasteur Drive, Stanford, California 943055327. email: neurokim@stanford.edu. 